\title{
PROGNOSTIC FACTORS IN THE EARLY POSTOPERATIVE OUTCOME OF ESOPHAGEAL ATRESIA. THE EXPERIENCE OF A TERTIARY CENTER OVER A 5 YEARS PERIOD
}

\author{
Mihaela Ciornei', Bogdan Savu', Elena Hanganu', Diana Ecaterina Popa ${ }^{1}$, \\ Marin Burlea ${ }^{2}$ \\ ${ }^{1}$ Pediatric Surgery Departament, \\ "Gr. T. Popa" University of Medicine and Pharmacy, Iasi \\ ${ }^{2}$ Pediatrics Departament, "Gr. T. Popa” University of Medicine and Pharmacy, Iasi
}

\begin{abstract}
Purpose. The aim of this study was to determine the influence of prognostic factors on the postoperative outcome of esophageal atresia patients, taking into consideration loco-regional particular features.

Methods. A retrospective analysis of the medical records of 28 patients diagnosed with esophageal atresia was conducted, using a logistic regression model. Patients had been admitted in our hospital between 2009-2014. The survival rate was recorded separately from the independent factors, such as demographic features, weight at birth, the timing of surgical treatment, co-morbidities, postoperative prognosis and management of complications.

Results. According to the Spitz prognostic classification, there were 21 patients in group I (birth weight over $1500 \mathrm{~g}$ with no major anomaly), 3 patients in group II (birth weight less than $1500 \mathrm{~g}$ or major cardiac anomaly) and 4 patients in group III (birth weight less than $1500 \mathrm{~g}$ plus major cardiac anomaly). The mortality rate was $33 \%$ in group I, $100 \%$ in group II and $100 \%$ in group III. The mean birth weight was $2282 \mathrm{~g} \pm 2 \mathrm{SD}$, and the mean gestational age was 31 weeks. The age at initial presentation was over 24 hours in 15 patients, with fatal outcome in 13 of them. The cardiac malformations presented as the associated anomalies with the highest risk. Surgical treatment was as follows: primary anastomosis in 21 cases, cervicostomy and gastrostomy in 6 cases, and Foker technique in 1 case.

Conclusions. The analysis of this series indicated a low survival rate for this pathology in our center. Besides the prognostic factors cited in literature (low birth weight and age at birth, associated cardiac malformations), we include, as risk factors for the increased mortality, the delayed diagnosis and presentation at our tertiary center. The further refinement of a multidisciplinary approach towards this pathology would contribute to a higher survival rate and an improved result of the therapeutic management.
\end{abstract}

Keywords: Esophageal atresia, survival rate, gap-length, anastomotic leak, demographic factors

\section{BACKGROUND}

Esophageal atresia and/or tracheo-esophageal fistula represent a congenital malformation with and incidence of 1 per 2500 births, being incompatible with life in the absence of surgical treatment. Improvements in neonatal intensive care, surgical techniques and the management of associated anomalies have determined an increase of survival rate of up to $90 \%$ in certain centers (1). The various proposed prognostic classification systems reflect this favorable outcome. In this study, we conducted a retrospective analysis of survival rates of patients treated in our tertiary center in Romania, using the data and prognostic factors cited in literature, keeping in mind the regional particularities. 


\section{METHODS AND MATERIALS}

Twenty eight patients included in our study were treated for esophageal atresia from 2009 to 2014. Respiratory, gastrointestinal and ano-rectal associated anomalies we treated surgically in the same center. Reviewing the medical documents, we gathered information regarding demographic data, birth weight, and anatomical classification of the esophageal malformation, age at surgical intervention, associated anomalies, surgical technique, outcome and management of complications, with the aim of identifying prognostic factors of successful surgical treatment.

Using univariate logistic regression analysis, the relationships between survival rates of targeted risk groups and the demographic parameters, physiopathological aspects and postoperative complications were studied. Data processing was achieved using IBM SPSS Statistic 20 program.

\section{RESULTS}

There were 19 boys and 9 girls, with a mean gestational age of 31 weeks and a mean birth weight of $2282 \mathrm{~g} \pm 2 \mathrm{SD}$. Fifteen newborns were aged over 24 hours at the initial presentation in the service, of which 11 died from sepsis and pulmonary aspiration lesions. One child was 6 days old at the time of presentation.

Over half of the children which had an anastomotic leak, were diagnosed and operated over 24 hours from birth. Two patients were not born in a medical care unit. Using the Spitz classification system, mortality in group I with 21 patients was of $33 \%$ and $100 \%$ in groups II and III with 3, and, respectively, 4 newborns (Table 1).

For the anatomical ranking of the malformation the Ladd classification (1) was used: 27 type III Ladd cases (esophageal atresia with distal fistula) and 1 case type IV Ladd (esophageal atresia with proximal and distal fistula). The last patient had been initially classified as a type III Ladd, but during a second surgical procedure for an anastomotic leak, the proximal fistula was identified and the classification was corrected. The distance between the esophageal segments and the tension at the site of the anastomosis had been evaluated using preoperative imaging investigations and by clinical observation during surgery. Six patients in the series had the gap between the esophageal ends larger than $3 \mathrm{~cm}$, thus ranging in the "long gap" category.

The cardiac malformations were the most frequent associeted, including 15 minor, non-cyanotic ones and 1 case with an anomaly at the point of emergence of the aortic artery, with fatal outcome within the first 24 hours. Ano-rectal malformations were associated in 4 cases and duodenal atresia in 2 cases. The initial treatment of ano-rectal anomalies consisted in performing a colostomy, simultaneously with the surgical correction of esophageal atresia in 3 cases and after 2 days, in the $4^{\text {th }}$ patient. Duodenal atresia was corrected concomitantly with the esophageal atresia, through a duodeno-duodenal anastomosis.

The period since the time of birth to the presentation in the tertiary center differed, based on the place of birth and proximity to the hospital. The surgical treatment was conducted in the first 24 hours, once the diagnosis was confirmed. Preoperative management included intravenous rebalancing, cardio-respiratory stabilization and aspiring of secretions from the proximal end. Patient evaluation included physical examination, as well as imaging (abdominal x-ray and abdominal, cardiac and transfontanellar ultrasonography).

Surgery was performed in 27 patients, the remaining newborn dying in the preoperative period from complications due to the severe cardiac anomaly. Surgical treatment included: primary anastomosis in 21 cases (through extra-pleural and right posterior lateral thoracotomy), cervicostomy and gastrostomy in 5 cases and Foker technique in 1 case (traction suture on the ends of the esophagus to reduce the distance and delayed anastomosis). A thoracic drainage system was placed in all patients, even though the approach was extra-pleural.

After the primary anastomosis, leakage was present in 6 cases, on an average of 7 days postoperatively. Four of these patients succumbed. In the patients in which cervicostomy and gastrostomy were the first intention procedures, 1 survival out of 5 was recorded. The patient treated with Foker technique presented with anastomotic leakage on the upper esophageal segment, requiring a cervicostomy, but had a fatal outcome.

Due to the hemorrhagic disease of the newborn, sepsis and multiple malformations, death occurred in 16 cases. Most patients had suffered fetal distress at birth. In 6 of the cases with death after the initial surgery, the anastomosis was found intact at post-mortem evaluation (Table 2). One patient treated with primary anastomosis developed a necrotizing enterocolitis 15 days postoperatively and required an ileostomy and a naso-gastric feeding tube. Thirteen days after this second surgery, the newborn had developed a gastric perforation. The outcome of trying to repair the defect was fatal. All 
complications occurring within one month from initial surgery were considered as Immediate postoperative ones. They consisted in anastomotic leaks, recurrence of fistula, gastric perforation, and suture detachment on the esophageal ends (9).

The initial management of anastomotic leakage involved placing a thoracic drainage tube, antibiotic therapy and feeding through a naso-gastric tube. If these control measures failed, an iterative thoracotomy was made to suture the leaking defect or to perform a cervicostomy and gastrostomy (in 4 cases). Postoperative results were evaluated using plain thoracic x-ray films and eso-gastro-duodenal radiographs with contrast media.

\section{DISCUSSIONS}

The survival rate is the lead parameter through which the quality of management in patients with esophageal atresia is measured. Throughout time, numerous classifications of prognostic factors were described (1-3). In 1962, Waterson and colleagues offered the first prognostic classification. Based on a retrospective study on 337 patients treated between 1980 and 1992 in the Hospital for Sick Children on Great Ormond Street in London, Spitz and colleagues showed that low birth weight and severe cardiac anomalies are influential factors in survival rates, if the neonatal intensive care and surgical techniques improve. Okamoto and colleagues have recently revised the Spitz classification system and devised a new one in which they state that in the context of an effective neonatal care of children with low and very low birth weight, severe cardiac anomalies should remain the sole prognostic factor of infants with esophageal atresia. Factors that independently influence the survival rate are, according to Poenaru et al (21), severe pulmonary anomalies which require preoperative mechanical ventilation. For the present analysis, the model of prognostic evaluation described by Spitz et al was used.

The data described in the literature recommend the initiation of surgical treatment after the first 24 hours, time period during which the cardio-vascular stabilization is obtained, excluding the respiratory distress syndrome that doesn't accelerate the timing of surgical intervention for fistula repair (1, $5)$. In the authors' center, all the admitted patients received an emergency surgical treatment, even though their respiratory condition hadn't required an emergency procedure.

Reviewing the Spitz classification, the survival rate in our study is lower than the one cited in lit- erature, $60 \%$ versus $93 \%$ in group I, with a $100 \%$ mortality rate in the rest of the groups. As parameters used to evaluate the outcome, besides low birth weight and age, we have included, as particular features: the time between the moment of birth and the initial presentation in the authors center and the delay in diagnosis. These parameters can lead to a higher risk of complications, such as aspiration pneumonia, hypoxic brain damage, infection with pathogenic bacteria and altered functional status.

No patient in the present study had been diagnosed antenatally. The prenatal diagnostic rates of this condition are between 30 and 50\%. Although the antenatal diagnosis doesn't improve the outcome, it offers a few advantages: the need of further evaluation for detecting associated malformations that can necessitate immediate surgical treatment (keeping in mind that esophageal atresia is not a surgical emergency), reduced time until initial presentation or birth programming close to a pediatric surgical unit and parental counseling to reduce the postpartum maternal anxiety. Lack of these logistic advantages represents a reflection of the insufficiency of territorial healthcare standards and of the quality of antenatal and neonatal care, that influence the morbidity and mortality of these children.

There are numerous case series in the literature that have described the evolution of patients with esophageal atresia through documenting the rate of complications, but few of the authors have tried identifying a direct relationship between the preoperative factors and the complications. The connection between the tension at the site of anastomosis and the anastomotic leak in the postoperative period was described by Kosloske et al (19). In the authors' center, this distance was measured radiologically (by counting the number of vertebrae between the esophageal ends), and intraoperatively, through direct examination (8). Anastomotic leak stands as the fiercest complication, as it can lead to a lifethreatening pneumothorax. There are current debates regarding the choice of conservative versus surgical approach in anastomotic leak scenario, with a tendency for the first option in most centers, by placing a thoracic drain, together with antibiotic and supportive treatment (parenteral nutrition and ventilation), until remission. There are no sufficient published data analyzing the long term outcome of these patients, including the risk of stricture development due to scar tissue, fibrosis around the anastomosis site, gastro-esophageal reflux, tracheomalacia and worsening of esophageal motility (17). 
In the study series, we recorded 6 anastomotic leaks, treated conservatively at first, but which ultimately required iterative thoracotomy, to suture the defect. In 5 of these cases, the evolution was unfavorable, imposing the performing of cervicostomy and gastrostomy. Postoperative incidence of anastomotic leak was $29 \%$, higher than in published studies (around 15-20\%) (7,8,15).

For various logistic reasons and taking into account the center's inexperience in minimal invasive approaches during the study period, thoracotomy was the preferred surgical approach. Literature statistics show that results after thoracoscopic approaches are still debatable, due to the high rates of complications and the existence of a steady learn curve (3).

Associated cardiac anomalies may cause problems in alimentation, with newborn fatigue during feeding; therefore a differential diagnosis needs to be made to discover the etiology of apnea (cardiac or respiratory). Except for 1 patient, no other children included in this study had life threatening major cardiac anomalies.

Ano-rectal anomalies as well as those of the gastro-esophageal tract (hypertrophic pyloric stenosis, duodenal atresia) may influence the postoperative evolution, as it happened in 6 of the patients in this study, death occurring rapidly in those with triple association (esophageal atresia, ano-rectal malformation and duodenal atresia).

Though birth weight and gestational age, the type of birth, the time until diagnosis, the immediate postnatal care are all unspecific factors, they may contribute to the status of these patients and should be considered prognostic factors in establishing mortality rates and the immediate development of complications.

\section{CONCLUSIONS}

The evaluation of this series clearly shows the occurrence of an upgrade of intensive neonatal care, anesthesia and performance of the surgical techniques in the authors' center. Nevertheless, the survival rates are still low. The results show that, due to the diagnostic delay and the late initial presentation in our center, the rise of mortality is obvious. The absence of an antenatal diagnostic suspicion, the poor pregnancy monitoring and the late presentation at medical centers may all lead to delivery occurring at hundreds of kilometers away from a tertiary center. The improvement of medical education of pregnant women, a correct management of the newly born which include the early detection of esophageal atresia, should be obtained through a multidisciplinary approach of this pathology, involving general practitioners, gynecologists, neonatologists and pediatric surgeons.

Complications prevention, improvement of survival rates and of long term outcomes, would require further investigations on implementing better surgical techniques, involving special instrumentation and materials (sutures, transanastomotic feeding tubes, drainage tubes). The evaluation of known prognostic factors in a prospective manner, keeping in mind the regional features, will allow a future decrease of postoperative morbidity and mortality in neonates with esophageal atresia.

\section{REFERENCES}

1. Spitz L. Oesophageal atresia. Orphanet J Rare Dis 2007; 2:24.

2. Haight C.T.H. Congenital atresia of the esophagus with tracheoesophageal fistula: extrapleural ligation of fistula and end to end anastomosis of esophageal ends. Surg Gynecol Obstet 1943; 76:672-88.

3. Lee S., et al, Thoracoscopic repair of esophageal atresia with tracheoesophageal fistula: Overcoming the learning curve, J Pediatr Surg (2014), http://dx.doi.org/10.1016/j.jpedsurg.2014.04.016

4. Bo Wang. et al, A nationwide analysis of clinical outcomes among newborns with esophageal atresia and tracheoesophageal fistulas in the United States, JSR 2014;190:604-612

5. Jones T.B., Kirchner S.G., Lee F.A., Heller R.M. Stomach rupture associated with esophageal atresia, tracheoesophageal fistula, and ventilatory assistance. AJR Am J Roentgenol 1980; 134:675.

6. Abdullah Alshehri, An analysis of early nonmortality outcome prediction in esophageal atresia. J Pediatr Surg 2012, 47, 881-884

7. Castilloux J., Noble A.J., Faure C. Risk factors for short and long-term morbidity in children with esophageal atresia. J Pediatr 2010; 156:755-60.

8. Jane McKinnon L.K.A. Prediction and prevention of anastomotic complications of esophageal atresia and tracheoesophageal fistula. J Pediatr Surg 1990;25:778.

9. Lewis Spitz, Esophageal atresia Lessons I have learned in a 40-year experience, J Peditr Surg, 2006, 41, 1635-1640

10. Sfeir R., Bonnard A., Khen-Dunlop N., et al. Esophageal atresia: data from a national cohort. J Pediatr Surg 2013;48:1664.

11. Torfs C.P., Curry C.J., Bateson T.F. Population-based study of tracheoesophageal fistula and esophageal atresia. Teratology 1995; $52: 220$

12. Holland A.J., Fitzgerald D.A. Oesophageal atresia and tracheooesophageal fistula: current management strategies and complications. Paediatr Respir Rev 2010;11:100. Quiz 106-7. 
13. Niramis R., Tangkhabuanbut P., Anuntkosol M., Buranakitjaroen V., Tongsin A., Mahatharadol V. Clinical outcomes of esophageal atresia: comparison between the Waterston and the Spitz classifications. Ann Acad Med Singapore 2013; 42:297.

14. Choudhury S.R., Ashcraft K.W., Sharp R.J., Murphy J.P., Snyder C.L., Sigalet D.L. Survival of patients with esophageal atresia: influence of birth weight, cardiac anomaly, and late respiratory complications. J Pediatr Surg 1999; 34:70. discussion 74.

15. Rui Zhao. The outcome of conservative treatment for anastomotic leakage after surgical repair of esophageal atresia, J pediatr Surg 2011, 46,2274-2278.

16. Su B.H., Hsieh H.Y., Chiu H.Y., et al. Nosocomial infection in a neonatal intensive care unit: a prospective study in Taiwan. Am J Infect Control 2007; 35:190-5.

17. Nasr A., Ein S.H., Gerstle J.T. Infants with repaired esophageal atresia and distal tracheoesophageal fistula with severe respiratory distress: is it tracheomalacia, reflux, or both? J Pediatr Surg 2005; 40:9013.

18. Tovar J.A., Fragoso A.C. Current controversies in the surgical treatment of esophageal atresia. Scand J Surg 2011; 100:273-8.
19. Lee H.Q., et al. Long-gap oesophageal atresia:comparison of delayed primary anastomosis and oesophageal replacement with gastric tube, J Pediatr Surg (2014), http://dx.doi.org/10.1016/j. jpedsurg.2014.09.017

20. Tatsuya Okamoto, MD, Shigeru Takamizawa, MD, Hiroshi Arai, MD, Yuko Bitoh, MD. Esophageal atresia: Prognostic classification revisited, J Pediatr Surg 2009; 145: 675-681

21. Dan Poenaru, Jean-Martin Laberge, Ian R. Neilson, Frank M. Guttman. A new prognostic classification for esophageal atresia, J Pediatr Surg 1991; 4:505

22. Charles Garabedian, Rony Sfeir, Carole Langlois, Arnaud Bonnard, Naziha Khen-Dunlop, Thomas Gelas, Laurent Michaud, Fréderic Auber, Fréderic Gottrand, Véronique Houfflin-Debarge. Does prenatal diagnosis modify neonatal treatment and early outcome of children with esophageal atresia? American Journal of Obstetrics and Gynecology, 2015, 212: 340.e1-340.e7 